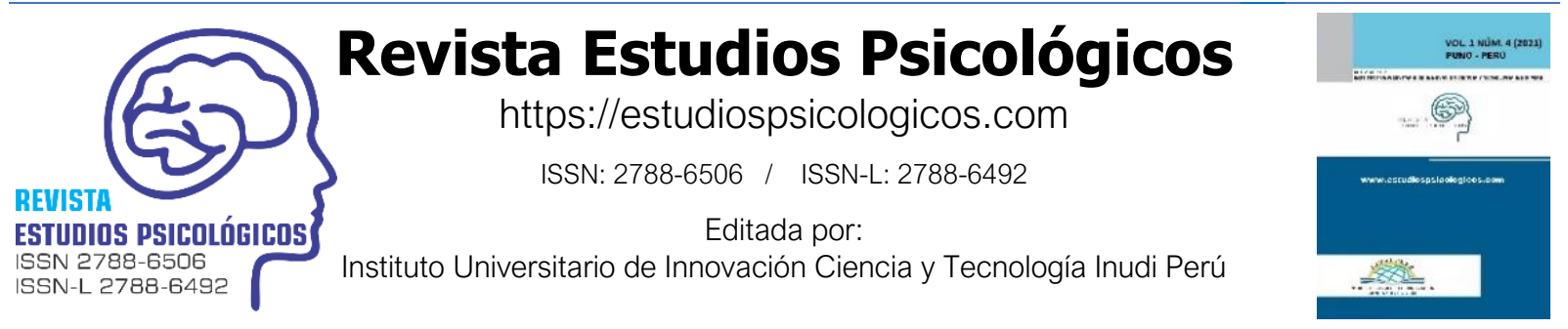

ARTÍCULO ORIGINAL

\title{
Componentes psicoemocionales y humanísticos dentro de la educación universitaria ecuatoriana: un análisis desde la docencia
}

\section{Psycho-emotional and humanistic components within Ecuadorian university education: an analysis from teaching}

Componentes psicoemocionais e humanísticos da educação universitária equatoriana: uma análise a partir do ensino

\author{
Leonardo Tarqui ${ }^{1}$ \\ Universidad Nacional de San Luis, Provincia de San Luis, Argentina \\ (iD https://orcid.org/0000-0001-9004-8988
}

D0I: https://doi.org/10.35622/j.rep.2022.01.002

Recibido 24/10/2021/ Aceptado 01/01/2022

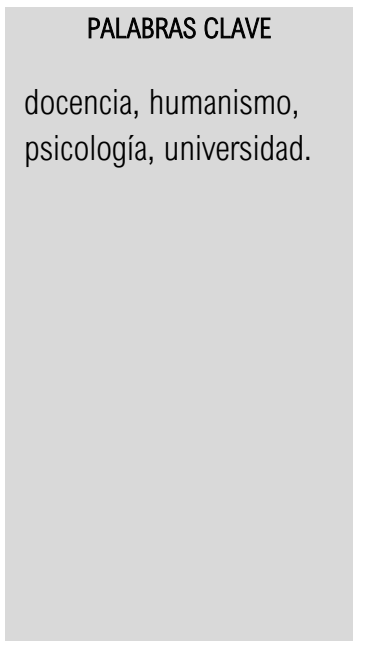

KEYWORDS

teaching, humanism, psychology, university.

\begin{abstract}
RESUMEN. El presente estudio fenomenológico hermenéutico, se fundamenta paralelamente en diversos elementos experienciales adquiridos a través del tiempo en universidades públicas y privadas de Ecuador. Se identificaron una serie de situaciones y fenómenos conductuales y psicoafectivos presentes en el alumnado, los cuales, influyen directamente sobre sus aspectos motivacionales, madurativos, deontológicos, inclusivos, y en general; socioculturales. Los mismos que deben ser abordados y subsanados, para que los futuros profesionales contribuyan en las distintas áreas de su especialidad, con total entrega, moral y ética dentro de su accionar, acoplándose al continuo engranaje evolutivo de la sociedad. Se mencionan algunos procesos y cambios experimentados en la educación superior en el país mitad del Mundo durante los últimos años. Hechos que evidentemente afectan a toda la estructura educativa, incluido el docente. Se destaca que la educación actual, debe contener sólidos fundamentos estructurales éticos, humanísticos, y con un elevado grado de compromiso orientado al servicio a la comunidad. En este camino, el docente deberá concientizar sobre los elementos psicoemocionales del alumno, mientras este lo permita, considerándolo en primera instancia como ser humano, y posteriormente como alumno.
\end{abstract}

ABSTRACT. The present hermeneutic phenomenological study is based on several experiential elements acquired over time in public and private universities in Ecuador. A series of behavioral and psycho-affective situations and phenomena present in the students were identified, which directly influence their motivational, mature, deontological, inclusive, and general aspects; sociocultural. The issues must be addressed and resolved, so that future professionals can contribute to the different areas of their expertise, with total delivery, morals, and ethics within their action, joining the continuous evolutionary gear of society. Some processes and changes experienced in higher education in the middle of the world have been mentioned in recent years.

\footnotetext{
${ }^{1}$ Correspondencia: leonardosicdeporte@yahoo.es
} 
Books that affect the entire educational structure, including the teacher. It is noteworthy that current education must contain solid ethical and humanistic structural foundations and a high commitment to community service. On this path, the teacher will have to raise awareness about the psychoemotional elements of the student, as long as this allows, considering him first as a human being, and later as a student.

\begin{tabular}{ll}
\hline PALAVRAS-CHAVE & RESUMO. 0 presente estudio fenomenológico hermenológico, se fundamentalmente paralelamente \\
ensino, humanismo, & en diversos elementos experienciales adquiridos a través del tiempo en universidades públicas y \\
psicologia, universidade. & $\begin{array}{l}\text { privadas de Ecuador. Se identificar uma série de situações e fenômenos condutivos e psicoafetivos } \\
\text { presentes no aluno, os alunos, influenciam diretamente sobre os aspectos motivacionais, } \\
\text { madurativos, deontológicos, inclusivos e em geral; socioculturales. Los mismos que deben ser } \\
\text { perfeitamente y subsanados, for that los futuros profesionales contribuyan en las distintas áreas de } \\
\text { su especialidad, con entrega total, moral y ética dentro de su accionar, acoplándose al continuo } \\
\text { engranaje evolutivo de la sociedad. Veja mencionan algunos procesos y cambios experimentados } \\
\text { en la educación superior en el pais mitad del Mundo durante los ultimos años. Hechos que } \\
\text { evidentemente afecta a toda a estructura educativa, incluido el docente. Se destaca que la educación } \\
\text { atual, debe contener sólidos fundamentos estructurales éticos, humanísticos, y un elevado grado } \\
\text { de compromiso orientado al servicio a la comunidad. Neste caminho, o docente deberá concientizar } \\
\text { sobre os elementos psicoemocionales do alumno, mientras este lo permitir, considerar a primeira } \\
\text { instanciação como ser humano, e posteriormente como alumno. }\end{array}$ \\
\hline
\end{tabular}

\section{INTRODUCCIÓN}

Dentro de la educación universitaria a nivel de Latinoamérica y el Caribe, entre las principales metas planteadas desde inicios del Siglo XXI, destaca el concretar la reducción de la brecha digital y social, acarreada desde décadas pasadas. Se tiene como prioridad, garantizar un avance y desarrollo igualitario de las personas a nivel del planeta, más aún, con los fenómenos de globalización en los que el ser humano se ve directamente afectado.

El planteamiento de Ramírez (2020) hace referencia a que la escuela tradicional, sufrió un cambio radical a raíz de la aparición de la COVID-19. La educación debió adaptarse en apenas cuestión de semanas, a nuevos procesos de enseñanza-aprendizaje, metodologías de intervención y evaluación docente. Incluso se obligó a los educadores a establecer espacios de capacitación sobre el manejo de Tecnologías de la Información y la Comunicación (TIC) y herramientas pedagógicas virtuales.

La anulación del sistema presencial en las aulas, generó una transformación y transferencia de programas y planes docentes a la modalidad online, convirtiéndose en un verdadero y continuo desafío para los docentes, incluidos los de nivel universitario; y también para los alumnos. Además, este fenómeno de aislamiento, conllevó al surgimiento de una serie de problemáticas psicosociales y emocionales, afectando inclusive, a la salud mental y física, pues existió un incremento del sedentarismo en las personas a nivel mundial (Ortega et al., 2021).

Es posible mencionar que durante esta etapa de transición a la educación en línea o virtual, se experimentó a nivel psicológico, una serie de afecciones emocionales, afectivas y de vínculos relacionales. Los procesos de enseñanza y aprendizaje tuvieron que recurrir, de acuerdo a la literatura académica, a softwares y plataformas 
tecnológicas como: Facebook, email, Classroom, Skype, Webex, YouTube streaming, Zoom, Google drive, Moodle, Microsoft Teams, LMS, Edtech, entre las principales, excluyendo los procesos relacionales presenciales (Bedoya et al., 2021).

Ozamiz et al. (2020) plantean que, de acuerdo a la bibliografía existente, en cualquier desastre biológico, como el que aún nos encontramos superando, existen elementos psicoemocionales que siempre estarán presentes, el miedo, la incertidumbre, vacíos afectivos. Es importante que los países mantengan programas de intervención médicos y psicológicos, instruyendo sobre los diferentes mecanismos de actuar. Se requiere diagnosticar y regular continuamente el estado psíquico de la población, ya que cada grupo social, puede percibir el fenómeno de forma diferente.

Una revisión bibliográfica en psiquiatría, elaborada por Paricio y Pando (2020) identifica que los desastres naturales se encuentran dentro de los eventos con potencial efecto traumático especificados en el Manual Diagnóstico y Estadístico de Enfermedades Mentales (DSM-5). Entre los principales trastornos e impactos de carácter mental, se mencionan el estrés postraumático, etapas de duelo, ansiedad, depresión. En el caso de los niños, tristeza, irritabilidad, y en algunos otros, duelo por separación. Esto debido a que sus cuidadores han tenido que ser aislados por cuarentena, o viceversa.

Un estudio reciente, sobre los efectos psíquicos de la pandemia, realizado en México, a nivel de la población universitaria en docentes y alumnos, reveló que existe un impacto sobre la salud mental de los dos grupos poblacionales, en los niveles de: depresión, ansiedad, insomnio, impacto del evento y agotamiento, siendo los más afectados, los educadores (Colín, 2021).

Para investigadores como Fernández (2020) el estado de ánimo, las emociones, sentimientos, y en general la salud mental, son determinantes durante los procesos de enseñanza-aprendizaje, a todo nivel, y en especial en la niñez y juventud. En este sentido, resalta el enorme esfuerzo, las dificultades y limitaciones que implica el distanciamiento obligatorio a nivel mundial dentro de los procesos educativos, afectando más, la crisis de la COVID-.19, a todos los elementos psicoemocionales citados inicialmente.

Una investigación observacional descriptiva de corte transversal en estudiantes de medicina, realizado por los Panizo et al. (2021) sobre la importancia de los valores y la responsabilidad como profesionales de la salud, en la cual, se aplicó un cuestionario que exploraba conductas morales y éticas durante la pandemia; puso en evidencia una serie de muestras de humanismo y mayor sensibilidad a la carrera médica y a las personas, esto luego de afrontar diferentes situaciones que se presentaron en la comunidad cubana durante la crisis de la COVID-19. 
Caicedo et al. (2021) presentan una clara visión, en la cual, queda en evidencia que la universidad pública de Ecuador, a pesar de haber experimentado en los últimos años una serie de drásticas reducciones al presupuesto del Estado, destinado a la educación universitaria; se encuentra obligada a innovarse y acoplarse a los distintos comportamientos, alternativas y metas, que presenta la contemporaneidad. Busca mantener su desarrollo y misión, deja atrás, las prácticas pedagógicas del pasado, busca la formación integral del alumno, formándolo con elementos humanísticos.

Un reportaje presentado por el Diario Digital ecuatoriano Primicias (2020) indica que la última afección presupuestaria en el año 2019 a las universidades públicas del Ecuador, fue alrededor de USD 105,4 millones, de USD 931 millones presupuestados, y a consecuencia de los recortes, existió un nuevo replanteamiento a USD 833,6 millones, es decir, USD 97,6 millones menos de lo planteado inicialmente. La asignación para gastos oscilaba entre 17 millones de dólares, pero con la revisión presupuestaria, quedaron 9,3 millones; y en el 2020, la preasignación por gratuidad estaba prevista en 183 millones. A esto se suma la Ley Reformatoria para la Equidad Tributaria de Ecuador, la cual dispone que las universidades públicas, así como las cofinanciadas, perciban una compensación, por la llamada donación del Impuesto a la Renta.

En base a este contexto introductorio, resalta la importancia de los factores psicológicos, humanísticos y pedagógicos dentro de la educación universitaria, además que, deberán ser adaptados, actualizados, y aplicados por los docentes. De esta forma se podrá potenciar todas las capacidades intelectuales de sus alumnos, incluyendo sobre manera, el desarrollo de elementos éticos, morales y humanos, garantizando que su actuación futura como profesional, contribuya de manera íntegra a la sociedad, con elevados conceptos altruistas.

\section{MÉTODO}

El método aplicado durante la presente exploración, fue de carácter fenomenológico hermenéutico. Se caracteriza por la descripción e interpretación de las experiencias vividas, procedimiento destacado a nivel de las ciencias de la pedagogía, psicología y sociología (Fuster, 2019).

A nivel del proceso metodológico documental, se priorizó la exploración de la información, e identificación de la cadena de búsqueda correcta (Barros \& Turpo, 2017). De acuerdo a este parámetro, la presente investigación se la realizó en base a una exhaustiva búsqueda en las bases de datos Psycinfo, MEDLINE, Google Académico, Psicodoc y PsycARTICLES. Las palabras claves empleadas fueron: "universidad”, "psicología”, "humanística”, y "docencia". Para ampliar los resultados de la búsqueda, el término "salud mental" fue sustituido por su traducción inglesa "university" y "psychology" por "humanistics", así como la palabra "docencia" tomó la forma de "teaching". 


\section{RESULTADOS Y DISCUSIONES}

El trabajo propuesto por Polaino et al. (2020) sobre la aplicación de modelos pedagógicos en la universidad ecuatoriana, especifica que lo primordial dentro de este sistema, es contar con docentes con elevados niveles de preparación en la parcela científica, cultural y humanística. Esto permitirá fusionar lo académico, la investigación, y los proyectos de vinculación con la sociedad, los mismos que sensibilizan al estudiante de grado y postgrado a nivel de servicio a la colectividad. Son además su primer acercamiento a elementos morales y de valores, referentes a las distintas profesiones.

Un estudio realizado en estudiantes de la carrera de Derecho de la Universidad de los Andes UNIANDES del País Mitad del Mundo, destaca la importancia del factor humanístico, el cual, debe ser integrado dentro de los contenidos de los cursos teóricos, y en las practicas a nivel de la especialidad. Estos permiten adecuadas condiciones en el plano psicológico y pedagógico, para que el docente se desarrolle en un ambiente moralmente positivo. En el caso específico de estos profesionales, permite la formación de abogados con sólidas creencias morales, orientados humanísticamente al servicio a la sociedad (Paronyan et al., 2018).

Otro de los elementos trascendentales dentro de la educación a nivel mundial, y evidentemente en Ecuador, gira en torno a la discriminación e inclusión de la mujer dentro de la academia. Mantilla y Zamora (2017) en un estudio realizado en la Universidad Técnica de Ambato, menciona la influencia de la marginación, y su efecto sobre la autoestima en el sexo femenino, a tal punto de condicionar la elección de sus estudios, el papel activo o pasivo, a protagonizar en la sociedad a la que pertenece.

Una investigación experimental, a nivel de los sesgos de carácter cognitivo y elementos de percepción de bienestar en la población universitaria ecuatoriana, expone que hombres y mujeres, mantienen puntuaciones adaptativas equilibradas en lo que respecta a un bienestar subjetivo y psicológico, siendo el sexo masculino quienes presentan inclinaciones al pesimismo. Todo esto se encuentra sujeto a factores sociales, económicos, políticos, familiares, lo cual, es un estudio netamente referencial (Torres et al., 2020).

Palacios et al. (2017) refieren que los valores tienden a variar de una cultura a otra, siendo dependientes y moldeables en base a factores económicos, geográficos, religiosos, con influencias directas de elementos socioculturales adquiridos, como las costumbres, creencias, e idiosincrasias de una región o comunidad. En este marco conceptual, el autor manifiesta la urgente necesidad de una educación en valores a nivel de todas las estructuras educativas, quizá con más énfasis en la población universitaria de Ecuador, pues existe una posible pérdida de elementos morales y éticos, en la sociedad actual del país tricolor. 
Dentro de estos procesos de enseñanza-aprendizaje en valores en estudiantes ecuatorianos, de manera interesante, el trabajo realizado por Morales et al. (2017) incluye otro elemento primordial; la formación en el cuidado y respeto por el medio ambiente. Este inicia desde el barrio, hasta los escenarios más intrincados de la sociedad, promueve estilos de vida sostenible y conservación de la humanidad, más aún, en la presente realidad consumista. Urbina et al. (2020) exploran la relación existente entre los diferentes estilos de religiosidad, y los valores en estudiantes universitarios en la región costera de Ecuador, específicamente en Esmeraldas. Se encuentran determinados elementos fundamentales en la formación de aspectos morales, como la raza, la historia cultural, el sexo, la zona geográfica, y factores extrínsecos e intrínsecos.

Entre los aspectos más destacados dentro la presente búsqueda teórica, Suárez et al. (2019) de manera muy valiosa, refiere que la formación en valores y el enfoque humanístico dentro de la universidad ecuatoriana, supone un fuerte compromiso entre las distintas instituciones de educación superior, profesores, el papel activo del estudiante, y la visión e intervención a través de acciones inclusivas, que el Estado provea. Esto como primera estructura reguladora de políticas sociales y públicas.

García et al. (2021) plantean la necesidad de una adecuada preparación del docente frente a los procesos educativos virtuales, guiados bajo una ética humanística, esto fundamentado en experiencias de docencia en Ecuador durante la crisis de la COVID-19. Reluce la importancia del uso adecuado de pedagogías virtuales, pues los profesores, a raíz de la crisis mundial, son "migrantes digitales”. Se deben actualizar y explorar metodologías de intervención propicias, que mantengan el dinamismo y atención del alumnado, aparte de no descuidar los elementos humanísticos y psicoemocionales de los mismos.

Finalmente, y dentro del contexto ecuatoriano, López et al. (2016) identifican la determinación de un correcto accionar dentro de los procesos de vinculación con la colectividad, pues a partir de este involucramiento con el entorno, se facilita la interacción cultural de valores morales y éticos, psicológicos, emocionales, y en general, un total enriquecimiento mutuo que se convierte en el "entrenamiento real" de los futuros profesionales.

\section{DISCUSION}

Espinoza y Guachamín (2017) plantean que un componente esencial para que las entidades de educación superior fundamenten su accionar en base a políticas estructuradas y aplicables en la formación de valores, 
ética, y con un elevado sentido de humanismo, radica en la responsabilidad social universitaria (RSU). La cual consiste en una filosofía, la misma que debe ser arraigada e interiorizada a nivel grupal e individual, dentro de estas entidades formativas a nivel cultural. Esto permitirá una actuación responsable, capaz de generar diversos impactos educativos, laborales, cognitivos, ambientales, y en general sociales, promoviendo un crecimiento humano comprometido y propicio.

Existen también otras perspectivas conceptuales, como las que plantean Reyes et al. (2017) donde resaltan que una educación integral universitaria, para alcanzar los estándares formativos en valores, respeto a las normas sociales, un accionar ético, un alto nivel de patriotismo, identidad nacional, y responsabilidad con el entorno; se ve influenciada por el componente político y normativo de cada sociedad. Este determinante, dentro de todo el contexto que rige y regula a la sociedad, específicamente en el caso de Ecuador, "El Plan Nacional del Buen Vivir", es un valioso marco organizador y motivador a estas valiosas prácticas.

Desde el enfoque de Vallejo et al. (2021) para que exista una educación universitaria con un enfoque equitativo ético y humanístico; es primordial en prima instancia, una actualización de los diseños curriculares de las distintas carreras en Ecuador. A esto se suma, la capacitación continua de los docentes, para fortalecer las capacidades comunicativas, su correcta actuación en el plano docente, investigativo y de vinculación. Es trascendental que se motive al estudiante en niveles formativos más allá de los sílabos, desarrollando su pensamiento crítico, innovador, creativo, con elevados elementos éticos y humanísticos, planteándole una visión más amplia del mundo.

Otro elemento que deben considerar las instituciones de educación superior, es el factor psicoemocional de los estudiantes, siendo uno de los indicadores, la población estudiantil con bajo rendimiento académico. Un estudio realizado en la Pontificia Universidad Católica del Ecuador en estudiantes de las carreras de psicología clínica y organizacional, indica la importancia de la exploración e intervención psicológica del estudiantado. Esto corresponde a competencias de las áreas que integran bienestar estudiantil, como especialistas, son quienes deberán intervenir direccionando sus acciones y de los profesionales que conforman esta estructura tan importante dentro de las entidades de educación superior, donde se vele por factores familiares, económicos y sociales de los alumnos (Lascano \& Moreta, 2019).

Existen otros elementos de carácter cultural que se encuentran relacionados directamente con el bienestar, psiquismo y la salud del sector poblacional estudiantil. Boira et al. (2017) en su estudio dentro de tres universidades ecuatorianas, explora las diferentes distorsiones cognitivas, comportamientos violentos, y acciones de violencia contra la mujer, esto a nivel de parejas estudiantiles. En su investigación, encontraron que existe un mayor sexismo hostil por parte de los hombres; sin embargo, los datos son alarmantes por el nivel 
de violencia contra la mujer. Esto dentro del ambiente académico, siendo factores que demuestran la necesidad de una reeducación en valores, a pesar de ser una población explorada, que busca una preparación de tercer nivel.

Vega y Borrero (2021) realizan una exploración de carácter psicológico en los estudiantes de psicología de la Universidad Técnica de Manabí, identificando la importancia de los elementos resilientes que dispone la personalidad de cada individuo. En este caso, de cada alumno, se relaciona que, a mayor nivel de resiliencia, existe una mejor disposición para enfrentar las adversidades, indicando menores porcentajes en la escala de depresión. Esto refleja que, los factores psicológicos del alumnado, se encuentran en dependencia de las características individuales, y del contexto muy personal de cada individuo.

\section{CONCLUSIONES}

Entre las principales conclusiones obtenidas durante la presente investigación, se identifica en primer lugar, la necesidad de un fortalecimiento de la educación ecuatoriana a nivel de valores, normas éticas, y un elevada "dosis" de humanismo en las aulas, no únicamente dirigido al sector estudiantil, también orientado al cuerpo docente y administrativo. Esto permitirá generar una cultura universitaria fundamentada en sólidos elementos altruistas y de virtudes.

Otro elemento que resalta, es la influencia de la sociedad en la cual se encuentra inmiscuido el estudiante, todo su entorno, el cual lo envuelve, y, en definitiva, le otorga conceptos y estereotipos conductuales de su accionar, impregnándole normas, ideas, valores, evidentemente, estos también son adquiridos en las primeras estructuras formativas; como el hogar, la escuela y el colegio. Este bagaje experiencial, sale a la luz en la universidad, donde existe un mayor rose y exigencias sociales. Por tal motivo, en algunos casos es necesario realizar un proceso reeducativo o reestructuración de criterios ligados a un comportamiento correcto dentro de la sociedad, normas éticas en su profesión, y regulación de conductas, como la violencia contra la mujer, el cuidado del medio ambiente, y otras normas transcendentales e imperativas.

Finalmente, los factores psicológicos del estudiante y del profesor, juegan un rol imprescindible dentro de los procesos de enseñanza-aprendizaje, es fundamental que exista una exploración y seguimiento de los educandos, más aún dentro de las entidades de educación universitaria pública de Ecuador. En base a los estudios explorados, y en factores vivenciales, se menciona que existen alumnos que provienen de sectores marginales, otros que presentan deficiencias culturales, producto de una baja educación académica en las estructuras de primaria y secundaria. 
Es importante generar adicionalmente, escuelas formativas y talleres dentro de la universidad, donde se aborden temáticas como el machismo, la violencia contra la mujer, temas de prostitución, drogodependencias, autoestima, campañas en vinculación con estructuras del Estado, como Derechos Humanos, Ministerio de Salud Pública, Ministerio de Inclusión Económica y Social, y Consejo Nacional Para Discapacidades. De tal forma que se brinde una visión integral e inclusiva de la educación superior, comprometiendo a los diversos organismos estatales, que pueden reforzar una preparación académica de calidad.

\section{Conflicto de intereses / Competing interests:}

$\mathrm{El}$ autor declara que no incurre en conflictos de intereses.

\section{Rol de los autores / Authors Roles:}

No aplica.

\section{Fuentes de financiamiento / Funding:}

El autor declara que no recibió un fondo específico para esta investigación.

\section{Aspectos éticos / legales; Ethics / legals:}

El autor declara no haber incurrido en aspectos antiéticos, ni haber omitido aspectos legales en la realización de la investigación.

\section{REFERENCIAS}

Barros, C., \& Turpo, 0. (2017). La formación en el desarrollo del docente investigador: una revisión sistemática. Espacios, 38(45), 11. https://www.revistaespacios.com/a17v38n45/a17v38n45p11.pdf

Bedoya, C., Murillo, G., \& González, C. (2021). Gestión universitaria en tiempos de pandemia por COVID-19: análisis del sector de la educación superior en Colombia. Estudios Gerenciales, 37(159), 251-264. https://doi.org/10.18046/j.estger.2021.159.4409

Boira, S., Chilet, E., Jaramillo, S., \& Reinoso, J. (2017). Sexismo, pensamientos distorsionados y violencia en las relaciones de pareja en estudiantes universitarios de Ecuador de áreas relacionadas con el bienestar y la salud. Universitas Psychologica, 16(4), 1. https://doi.org/10.11144/Javeriana.upsy16-4.spdv

Caicedo, P., Durán, M., Becerra, J., \& Montilla, A. (2021). Los desafíos de la universidad pública ecuatoriana ante el escenario post COVID-19. ReHuSo: Revista de Ciencias Humanísticas y Sociales, 6(3), 29-39. https://doi.org/10.5281/zenodo.5512822

Colín, C. (2021). Impacto de la pandemia Covid-19 sobre profesores y estudiantes en escuelas de negocios en México. Revista del Centro de Investigación de la Universidad la Salle, 14(55), 81-102. https://doi.org/10.26457/recein.v14i55.2773

Espinoza, G., \& Guachamín, M. (2017). La responsabilidad social universitaria en Ecuador. Estudios de la 
gestión:

revista

internacional

de

administración,

https://revistas.uasb.edu.ec/index.php/eg/article/view/568

Fernández, A. (2020). 2020: Estudiantes, emociones, salud mental y pandemia. Revista Andina de Educación, 4(1), 23-29. https://doi.org/10.32719/26312816.2021.4.1.3

Fuster, D. (2019). Investigación cualitativa: Método fenomenológico hermenéutico. Propósitos y Representaciones, 7(1), 201. https://doi.org/10.20511/pyr2019.v7n1.267

García, N., Quevedo, N., \& Cañizares, F. (2021). La evaluación en entornos virtuales de aprendizaje bajo la ética humanista: experiencias en universidad de Ecuador. Revista Varela, 21(59), 86-96. http://revistavarela.uclv.edu.cu/index.php/rv/article/view/1210

Lascano, M., \& Moreta, R. (2019). Síntomas clínicos, subclínicos y necesidades de atención psicológica en estudiantes universitarios con bajo rendimiento. Revista Educación, 43(2), 24. https://doi.org/10.15517/revedu.v43i2.32239

López, R., Cedeño, G., Lescay, D., \& Mestre, U. (2016). Relevancia del proceso de vinculación con la sociedad en la formación de profesionales en instituciones de educación superior del Ecuador. Revista Órbita Pedagógica, 3(2), 61-82. http://refcale.uleam.edu.ec/index.php/enrevista/article/view/2301/1234

Mantilla, M., \& Zamora, R. (2017). La inserción de la mujer en la educación superior ecuatoriana: caso universidad técnica de Ambato. Conrado, 13(2), 12-29. https://doi.org/10.17151/rlee.2017.13.2.2

Morales, T., Machado, L., \& Suárez, N. (2017). Enfoque de formación en valores por el medio ambiente en estudiantes universitarios (UTI Ambato- UMET Machala) en el Ecuador. Revista Conrado, 13(1-ext), 132137.

Ortega, D., Rodríguez, J., \& Mateos, A. (2021). Educación superior y la COVID-19: adaptación metodológica y evaluación online en dos universidades de Barcelona. Revista Digital de Investigación en Docencia Universitaria, 15(1), e1275. https://doi.org/10.19083/10.19083/ridu.2021.1275

Ozamiz, N., Dosil, M., Picaza, M., \& Idoiaga, N. (2020). Niveles de estrés, ansiedad y depresión en la primera fase del brote del COVID-19 en una muestra recogida en el norte de España. Cadernos de Saúde Pública, 36(4). https://doi.org/10.1590/0102-311x00054020

Palacios, L., Rivadeneira, J., García, A., \& Aray, C. (2017). La ética del docente Una perspectiva axiológica del proceso de la educación superior en el Ecuador. ReHuSo: Revista de Ciencias Humanísticas y Sociales, 1 , 
51-64. https://revistas.utm.edu.ec/index.php/Rehuso/article/view/1246

Panizo, S., Santos, T., \& Molina, V. (2021). Habilidades investigativas y valores en estudiantes de Estomatología en el contexto de la COVID-19. Revista médica Multimed, 25(2). http://www.revmultimed.sld.cu/index.php/mtm/article/view/1964

Paricio, R., \& Pando, M. (2020). Salud mental infanto-juvenil y pandemia de Covid-19 en España: cuestiones y retos. Revista de Psiquiatría Infanto-Juvenil, 37(2), 30-44. https://doi.org/10.31766/revpsij.v37n2a4

Paronyan, H., Melendez, R., \& Alfaro, M. (2018). Componente humanístico y formación universitaria en estudiantes de Derecho de UNIANDES Santo Domingo. INNOVA Research Journal, 159-168. https://doi.org/10.33890/innova.v3.n11.2018.800

Polaino, C., Romillo, A., \& Muñoz, J. (2020). Modelo educativo-pedagógico integrado de la Universidad de Otavalo, Ecuador. Formación universitaria, 13(5), 115-128. https://doi.org/10.4067/S071850062020000500115

Primicias. (2020). Reducción de presupuesto de universidades ecuatorianas. Presupuesto del año 2020. https://www.primicias.ec/noticias/economia/corte-falla-a-favor-de-finanzas-por-presupuesto-deuniversidades/

Ramírez, M. (2020). Transformación digital e innovación educativa en Latinoamérica en el marco del Covid-19. Campus Virtuales, 9(2). http://uajournals.com/ojs/index.php/campusvirtuales/article/view/744

Reyes, G., Guevara, J., \& Bonne, N. (2017). Formación de valores en futuros docentes ecuatorianos. MEDISAN, 21(4), 448-454. https://www.medigraphic.com/pdfs/medisan/mds-2017/mds174i.pdf

Suárez, G., León, J., Morales, M., \& Curbeira, D. (2019). Modelo para la formación de valores en la universidad $\begin{array}{llll}\text { inclusiva. } & \text { Revista } & \text { Conrado, } & \text { 75-88), }\end{array}$ https://conrado.ucf.edu.cu/index.php/conrado/article/view/1045/1055

Torres, C., Moreta, R., Ramos, M., \& López, J. (2020). Sesgo Cognitivo de Optimismo y Percepción de Bienestar en una Muestra de Universitarios Ecuatorianos. Revista Colombiana de Psicología, 29(1), 61-72. https://doi.org/10.15446/.v29n1.75853

Urbina, A., Martínez, J., \& Foronda, A. (2020). Estilos de religiosidad y valores de universitarios y universitarias de Esmeraldas, Ecuador. Revista Electrónica de Psicología Iztacala, 22(1), 336-354. http://www.revistas.unam.mx/index.php/repi/article/view/75394 
Vallejo, A., Kou, J., Ramírez, J., Valdez, F., \& Ramírez, Lady. (2021). El acceso a la Educación Superior por una educación equitativa, ética y humanista. Recimundo, 5(4), 74-83. https://recimundo.com/index.php/es/article/view/1299\#

Vega, J., \& Borrero, C. (2021). Niveles de resiliencia y la presencia de síntomas depresivos en los estudiantes universitarios. Revista Ecuatoriana de Psicología, 4(9), 134-142. https://doi.org/10.33996/repsi.v4i9.58 